\title{
Clinical significance of TrkB expression in nasopharyngeal carcinoma
}

\author{
SHI-SHENG LI, JIA-JIA LIU, SHUANG WANG, QING-LAI TANG, \\ BING-BING LIU and XIN-MING YANG
}

\begin{abstract}
Department of Otolaryngology, Head and Neck Surgery, The Second Xiangya Hospital, Central South University, Changsha, Hunan 410011, P.R. China
\end{abstract}

Received August 3, 2013; Accepted October 2, 2013

DOI: $10.3892 /$ or.2013.2878

\begin{abstract}
Recent research has demonstrated that tropomyosin-related kinase B (TrkB) plays an important role in neuronal survival, differentiation and migration; yet, its specific role in human nasopharyngeal carcinoma (NPC) is unclear. To elucidate its role in NPC, we examined TrkB expression in NPC tissues and cell lines, and investigated the correlation between TrkB expression and prognosis in patients with NPC. Immunohistochemical analysis was performed on NPC specimens from 108 patients with follow-up information. Additionally, reverse transcriptase-polymerase chain reaction (RT-PCR) and western blot analyses were used to determine TrkB expression levels in NPC and noncancerous nasopharyngeal tissues. RT-PCR and western blot analyses were also used to determine TrkB expression levels in 7 NPC cell lines and a nasopharyngeal epithelium cell line. High TrkB expression was significantly correlated with T classification, lymph node metastasis and clinical stage, respectively. Patients with NPC who had high TrkB expression had reduced disease-free survival and overall survival when compared with patients who had low TrkB expression. Multivariate Cox regression analysis revealed that TrkB overexpression was an independent prognostic factor for patients with NPC. Furthermore, TrkB was overexpressed in NPC specimens and cell lines. TrkB expression levels were significantly increased in NPC specimens, and enhanced levels were correlated with a poor prognosis in patients with NPC. These findings suggest that TrkB may contribute to NPC progression and represent a novel prognostic indicator for patients with NPC.
\end{abstract}

Correspondence to: Dr Xin-Ming Yang, Department of Otolaryngology, Head and Neck Surgery, The Second Xiangya Hospital, Central South University, 139 Renmin Road, Changsha, Hunan 410011, P.R. China

E-mail: x16y2013@outlook.com

Key words: nasopharyngeal carcinoma, TrkB, prognosis, metastasis

\section{Introduction}

Nasopharyngeal carcinoma (NPC) is much more common in Southeast Asia and Southern China but is rare in most other parts of the world (1). This disease is managed primarily by radiotherapy with or without chemotherapy (2). Despite significant progress in the methods of conventional therapies such as radiotherapy and chemotherapy, the outcome has been disappointing. The 5-year survival rate is still approximately between 34 and 52\% (3). Both local recurrence and lymph node metastasis are major causes of death in NPC and are key factors affecting clinical outcome and prognosis (4). The recurrence and metastasis of NPC is a multi-step process that often involves many complex biological and pathologic events. Despite extensive clinical as well as basic research efforts, the mechanisms involved in NPC metastasis still remain undetermined. To improve the prognosis of NPC, it is important to identify additional reliable prognostic factors indicating recurrence and metastasis of NPC. The search for reliable markers to predict prognosis is important, as intensive adjuvant treatment is critical for patients with a predictable poor outcome.

The tropomyosin-related kinase B (TrkB) is a member of the Trk family, and functions as a receptor tyrosine kinase. TrkB, which has brain-derived neurotrophic factor (BDNF) as its primary ligand, plays an essential role in nervous system development, neuronal survival, differentiation and maintenance (5). Recent reports indicate that TrkB has an important function in tumor pathology. Several studies have shown that TrkB is oncogenic not only in neurogenic original tumors, but also in other tumors outside of the neural system. Overexpression of TrkB has been reported in a variety of cancers, including neuroblastoma, cancer of the lung, pancreas, stomach and ovaries and has been associated with more aggressive malignant behavior and a poor prognosis (6-10). Furthermore, activation of the BDNF/TrkB pathway in some cancers is implicated in inhibition of apoptosis, increased proliferation, promotion of invasion, and facilitation of tumor progression by lymphangiogenesis-associated metastasis (11). Therefore, TrkB may play an important role in the progression and invasion of malignant tumors. However, the role of TrkB in human NPC remains unknown. Thus, the objectives of the present study were to determine the expression patterns of TrkB in NPC specimens and different NPC cell lines to 
explore the correlation with clinicopathologic features and prognosis in patients with NPC.

\section{Materials and methods}

Patients and tissue samples. Specimens of tissues were obtained from 108 NPC patients and 14 nasopharyngitis patients who underwent nasopharyngeal biopsy at the Department of Otolaryngology, Head Neck Surgery, The Second Xiangya Hospital of Central South University (Changsha, China) from January 2002 to January 2004. The NPC biopsy specimens were from patients who were diagnosed with primary NPC, and the noncancerous nasopharyngeal biopsy samples were from patients who were suspected of having NPC originally, but afterwards were pathologically and clinically proven to be clear. There were 87 male and 21 female patients, and their median age was 56 years (range, 19-86 years). The diagnoses for all patients were confirmed by histopathologic examination. The NPC tissue specimens were histologically confirmed and classified according to WHO classification into keratinizing carcinoma (type 1, 8 cases), differentiated non-keratinizing carcinoma (type 2, 30 cases) and undifferentiated non-keratinizing carcinoma (type 3, 70 cases). All patients were re-staged according to the 2010 American Joint Committee on Cancer (AJCC) criteria. There were 2 cases in stage I (T1N0M0 2 cases), 12 cases in stage II (T1N1M0 2 cases, T2N0M0 6 cases, T2N1M0 4 cases), 33 cases in stage III (T1N2M0 6 cases, T2N2M0 12 cases, T3N0M0 6 cases, T3N1M0 1 case, T3N2M0 8 cases) and 61 cases in stage IV (T1N3M0 5 cases, T2N3M0 22 cases, T2N1M1 1 case, T2N2M1 1 case, T2N3M1 2 cases, T3N3M0 13 cases, T3N2M1 1 case, T3N3M1 2 cases, T4N0M0 2 cases, T4N1M0 2 cases, T4N2M0 1 cases, T4N3M0 4 cases, T4N1M1 1 case, T4N2M1 3 cases and T4N3M1 1 case). Demographic details and tumor characteristics are listed in Table I.

All tissue specimens were fixed in $10 \%$ neutralized formalin and embedded in paraffin. Seventeen cases of NPC tissues among the 108 cases and 14 cases of noncancerous nasopharyngeal tissues were cut into 2 portions. One portion was fixed in $10 \%$ neutralized formalin and embedded in paraffin blocks for immunohistochemistry. The other portion was frozen immediately and stored in liquid nitrogen for RNA and total protein extraction.

The study was approved by the Central South University Ethics Committee. Informed consent was obtained from all of the patients. All specimens were handled and made anonymous according to the ethical and legal standards.

Treatment and follow-up. All patients received a complete course of radiotherapy with or without chemotherapy. Irradiation was administered primarily with megavoltage (6-10 MV) X-rays from a linear accelerator. The dose was 1.8-2.0 Gy/fraction, with 5 fractions/week. All patients were evaluated by nasopharyngoscope at 10-14 weeks after completion of radiotherapy. Patients were followed up regularly after treatments. The follow-up period was defined as the interval between the date of treatment completion and the date of either the patient's death or the last follow-up. Deaths were treated as censored cases. Clinical examinations, including an indirect mirror examination of the nasopharynx with or without
Table I. Clinicopathological features of the studied 108 cases of nasopharyngeal carcinoma (NPC).

\begin{tabular}{lc}
\hline Variables & No. of patients $(\%)$ \\
\hline Gender & \\
Male & $87(80.6)$ \\
Female & $21(19.4)$ \\
Age (years) & \\
$\leq 50$ & $56(51.9)$ \\
$>50$ & $52(48.1)$ \\
Histological type & \\
1 (keratinizing) & $8(7.4)$ \\
2 (differentiated non-keratinizing) & $30(27.8)$ \\
3 (undifferentiated non-keratinizing) & $70(64.8)$ \\
T classification & \\
T1 & $15(13.9)$ \\
T2 & $48(44.4)$ \\
T3 & $31(28.7)$ \\
T4 & $14(13.0)$ \\
N classification & \\
N0 & $16(14.8)$ \\
N1 & $11(10.2)$ \\
N2 & $33(30.6)$ \\
N3 & $48(44.4)$ \\
M classification & \\
M0 & $96(88.9)$ \\
M1 & $12(11.1)$ \\
Clinical stage & \\
I & $2(1.9)$ \\
II & $12(11.1)$ \\
III & $33(30.6)$ \\
\hline & $61(56.4)$ \\
\hline
\end{tabular}

a nasopharyngoscope, were performed during subsequent follow-ups. Chest X-ray, liver echo, whole body bone scans, computed tomography and magnetic resonance imaging (MRI) were performed only when clinically indicated. All diseasefree patients had a minimum follow-up of 60 months after the completion of treatment. Locoregional or distant recurrence was confirmed by histopathological or radiological criteria.

Immunohistochemistry. Specimens were obtained before therapy, fixed in $10 \%$ neutral buffered formalin, and embedded in paraffin. Serial $5-\mu \mathrm{m}$ sections were prepared. Slides were deparaffinized in xylene, rehydrated in graded alcohol, and incubated in $0.3 \%$ hydrogen peroxide in methanol to quench the endogenous peroxidase activity for $30 \mathrm{~min}$. The sections were subjected to microwave heat-induced antigen retrieval in

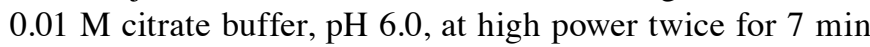
each. Nonspecific binding sites were blocked by a $20-\mathrm{min}$ incubation with normal horse serum.

The sections were incubated for $2 \mathrm{~h}$ at room temperature with primary rabbit polyclonal antibody detecting TrkB 
(1:100) followed by the addition of HRP-labeled goat antirabbit polymers. The streptavidin-biotin-peroxidase complex tertiary system (Boster, Wuhan, China) was used according to the manufacturer's instructions. All slides were visualized by applying 3,3'-diaminobenzidine tetrahydrochloride for $2 \mathrm{~min}$ and then counterstained with hematoxylin. Negative controls were non-immune rabbit $\operatorname{IgG}$ at the same dilution as for the primary antibody.

Sections were evaluated in a blinded manner and scored by two independent experienced pathologists. Expression levels of TrkB protein were assessed by observing the incidence and staining intensity (i.e., color strength) of immuno-positive cells. Staining intensity was scored as: 0 , negative; 1 , weak; 2 , strong. The incidence of positive cells was scored as: $1,0-50 \%$; $2,51-75 \% ; 3>75 \%$. Tumors were categorized into three groups based on the final staining point: low expression (scored 0-3), and high expression (scored 4-6) (11).

Cell culture. The Epstein-Barr virus (EBV)-negative, highly differentiated NPC cell line HNE-1 and the EBV-negative, lowly differentiated NPC cell line HNE-2 were established by the Cancer Research Institute, Xiangya School of Medicine, Central South University (12). The NPC cell line C666-1 which consistently expressed EBV was established from undifferentiated nasopharyngeal carcinoma by the Department of Anatomical and Cellular Pathology, Prince of Wales Hospital, University of Hong Kong (Hong Kong, China) (13). The NPC cell lines CNE-1 and CNE-2 were established from the primary tumors of patients with well-differentiated squamous cell NPC and poorly differentiated squamous cell NPC, respectively, by the Chinese Academy of Medical Sciences. The highly metastatic NPC cell line 5-8F and the nonmetastatic NPC cell line 6-10B were established from the NPC cell line SUNE-1 by the Cancer Research Institute of Sun Yatsen University, Guangzhou, China (14). The above 7 NPC cell lines were kindly provided by Cancer Research Institute, Xiangya School of Medicine, Central South University. The nontransformed nasopharyngeal epithelium cell line (NP-69) was established from the nasopharynx of a human by the Department of Anatomy, Faculty of Medicine, University of Hong Kong (15). This cell line was purchased from the Cell Experiment Center of Shanghai, China. All cell lines were maintained as monolayer cultures in Dulbecco's modified Eagle's medium (DMEM)/F12 medium (1:1) supplemented with $10 \%$ fetal bovine serum (FBS), $100 \mathrm{IU} / \mathrm{ml}$ penicillin and $100 \mathrm{IU} / \mathrm{ml}$ streptomycin at $37^{\circ} \mathrm{C}$ in a humidified atmosphere with $5 \% \mathrm{CO}_{2}$.

Total RNA isolation and RT-PCR. Total RNA of the tissue specimens, the 7 NPC cell lines and the nontransformed nasopharyngeal epithelium cell line (NP-69) was isolated by Simply P Total RNA Extraction kit (Bioer Technology, Inc., Hangzhou, China). Total RNA $(1 \mu \mathrm{g})$ was reverse transcribed by High-Capacity cDNA Reverse Transcription kits (Applied Biosystems, Foster City, CA, USA) according to the manufacturer's instructions. The primers for TrkB and GAPDH were synthesized by Sangon Biological Engineering Technology and Services Co., Ltd. (Shanghai, China) as follows: TrkB F, 5'-AGTCGCAGATGCTGCATATTAG-3' and R, 5'-TAGAA TGTCCAGGTAGACC-3'; GAPDH F, 5'-CTGCAGCATC
TTCTCCTTCC-3' and R, 5'-CAAAGTTGTCATGGATG ACC-3'. The reverse transcription product $(1 \mu \mathrm{l})$ was amplified by PCR using the following conditions: $95^{\circ} \mathrm{C}$ for $5 \mathrm{~min}$; followed by 32 cycles at $95^{\circ} \mathrm{C}$ for $30 \mathrm{sec}, 58^{\circ} \mathrm{C}$ for $30 \mathrm{sec}$, and $72^{\circ} \mathrm{C}$ for $30 \mathrm{sec}$, and an extension at $72^{\circ} \mathrm{C}$ for $5 \mathrm{~min}$. The PCR product $(8 \mu \mathrm{l})$ was then electrophoresed on $1.5 \%$ agarose gel. TrkB mRNA expression levels were quantified by Quantity One software (Bio-Rad Technical Service Department, Hercules, CA, USA) and represented as the densitometric ratio of the targeted gene to GAPDH. PCR experiments were repeated three times.

Western blot analysis. Total protein lysates were harvested from the tissue specimens and cell lines by lysis buffer $(20 \mathrm{mM}$ $\mathrm{Na}_{2} \mathrm{PO}_{4}, 150 \mathrm{mM} \mathrm{NaCl}, 1 \%$ Triton $\mathrm{X}-100,1 \%$ aprotinin, $1 \mathrm{mM}$ phenylmethylsulfonyl fluoride, $100 \mathrm{mM} \mathrm{NaF}$ and $2 \mathrm{mM}$ $\mathrm{Na}_{3} \mathrm{VO}_{4}$ ). Proteins $(50 \mu \mathrm{g})$ were separated by polyacrylamide gel electrophoresis on a $10 \%$ sodium dodecyl sulfate-polyacrylamide gel and transferred to polyvinylidene difluoride membranes. Membranes were incubated with the primary antibody overnight at $4^{\circ} \mathrm{C}$. On the next day, the membranes were washed with phosphate-buffered saline (PBS) three times and then the membranes were incubated with horseradish peroxidase-conjugated goat anti-rabbit secondary antibodies (all the antibodies were obtained from Santa Cruz Biotechnology, Inc., Santa Cruz, CA, USA). Finally, the proteins were detected by enhanced chemiluminescence (ECL) procedure. TrkB protein expression levels were quantified by Quantity One software and represented as the densitometric ratio of the targeted protein to $\beta$-actin. Western blot experiments were repeated three times.

Statistical analysis. Data are expressed as means \pm SD. Student's two-tailed t-test was used to compare TrkB expression between cancer and noncancerous nasopharyngeal tissues. The protein expression and clinicopathological parameters were compared using the $\chi^{2}$ test. Survival analysis was undertaken using the Kaplan-Meier method, and curves were compared using the log-rank test. Multivariate analysis, using the Cox's proportional hazards model, was applied to identify which factors were independent indicators for prognosis. All statistical evaluations were performed using the Statistical Package for the Social Sciences version 10.0 software program (SPSS Inc., Chicago, IL, USA). All tests were two-tailed, and a P-value of $<0.05$ was considered to indicate a statistically significant result.

\section{Results}

Results of immunohistochemistry and correlations between TrkB expression and clinicopathologic variables. The 108 NPC tumor samples were initially tested for TrkB protein expression by immunohistochemistry, and then the level of TrkB protein expression was correlated with clinicopathological parameters. Positive TrkB immunostaining was predominantly diffusely distributed throughout the cytoplasm of NPC cells (Fig. 1). Of all the samples tested, $65(60.2 \%)$ cases displayed low TrkB protein expression (Fig. 1C and D) and 43 (39.8\%) cases displayed high TrkB protein expression (Fig. 1E and F). The $\chi^{2}$ test showed that the TrkB expression level was significantly 

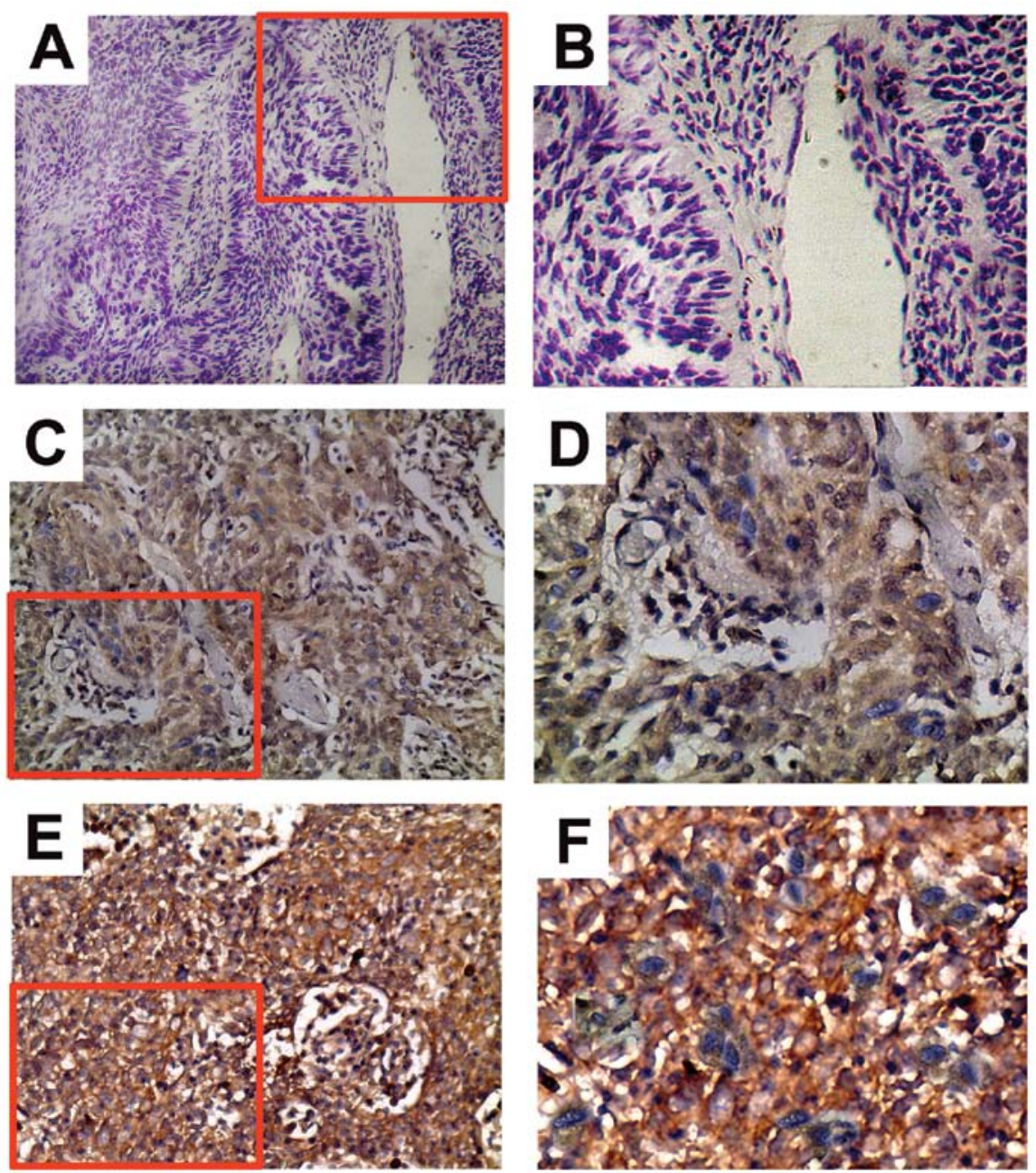

Figure 1. Immunohistochemistry of TrkB in human NPC tissues. (A and B) Negative control of TrkB in NPC tumor tissues. (C and D) Low expression of TrkB in NPC tumor tissues. (E and F) High expression of TrkB in NPC tumor tissues. (Original magnification in A, C and E, x100; original magnification in B, D and $\mathrm{F}, \mathrm{x} 400$ ).

associated with NPC tumor size (T classification) $(\mathrm{P}=0.015)$, lymph node metastasis ( $\mathrm{N}$ classification) $(\mathrm{P}=0.009)$, AJCC clinical stage $(\mathrm{P}=0.007)$. However, we did not find a significant association between TrkB expression level and age, gender and distant metastasis ( $\mathrm{M}$ classification). The relationship between clinicopathological characteristics and TrkB expression level in individuals with NPC are summarized in Table II.

Elevated expression levels of TrkB $m R N A$ and protein in NPC tissues. Seventeen freshly collected clinical NPC tissues and 14 freshly collected normal nasopharyngeal mucosal tissues were further investigated by RT-PCR and western blot analysis. The results of RT-PCR showed that the TrkB relative mRNA expression level was upregulated in the NPC tissues when compared to that in the nasopharyngeal mucosal tissues $(1.1 \pm 0.64$ vs. $0.38 \pm 0.27, \mathrm{P}=0.000<0.01$; Fig. $2 \mathrm{~A}$ and $\mathrm{C})$. Consistent with the mRNA data, the TrkB relative protein expression level in the NPC tissue was significantly higher when compared with that in the nasopharyngeal mucosal tissues $(0.52 \pm 0.24$ vs. $0.26 \pm 0.18, \mathrm{P}=0.003<0.01$; Fig. $2 \mathrm{~B}$ and $\mathrm{C})$.

Expression of TrkB mRNA and protein in NPC cancer cell lines. In previous experiments, we found that both TrkB mRNA and protein expression levels were upregulated in NPC tissues and were correlated with a number of clinicopathological parameters. The expression pattern of TrkB in human NPC cell lines was further investigated by RT-PCR and western blot analysis to verify the role of TrkB in vitro. As shown in Fig. 3, both mRNA and protein expression of TrkB in all the 7 NPC cell lines was significantly higher when compared to that in the normal nasopharyngeal epithelium mucosal NP-69 cell line. Moreover, compared to the other NPC cell lines, the 5-8F cell line, which has high metastatic potential, displayed the highest TrkB mRNA and protein expression.

Correlation between TrkB expression and prognosis. During the follow-up period of 5 years, 6 patients were lost to followup or died from other causes. Fifty-five patients were still alive without recurrence, and the remaining 47 patients had recurrent tumors. Thirty-nine of these 47 patients with recurrent disease died of locoregional recurrence or distant metastases within 5 years.

According to the TrkB protein expression levels, 108 patients were divided into 2 groups: 65 patients in the low expression group and 43 patients in the high expression group. In the Kaplan-Meier patient survival analysis, NPC 
Table II. Correlation between TrkB expression and clinicopathological variables in patients with NPC.

Variables

Gender

Male

Female

Age (years)

$\leq 50$

$>50$

Histological type

1 (keratinizing)

2 (differentiated non-keratinizing)

3 (undifferentiated non-keratinizing)

$\mathrm{T}$ classification

\section{$\mathrm{T} 1+\mathrm{T} 2$}

$\mathrm{T} 3+\mathrm{T} 4$

$\mathrm{N}$ classification

$\mathrm{N} 0+\mathrm{N} 1$

$\mathrm{N} 2+\mathrm{N} 3$

$\mathrm{M}$ classification

M0

M1

Clinical stage

I+II

III+IV
TrkB low expression, $\mathrm{n}$

Total
TrkB high expression, $\mathrm{n}$

$(n=43)$

P-value

87

21

56

52

8

30

70

63

45

27

81

96

12

14

94
56

9

33

32

4

15

46

44

21

22

43

60

5

13

52
31

12

23

20

4

15

24

19

24

5

38

36

7

1

42
0.071

A

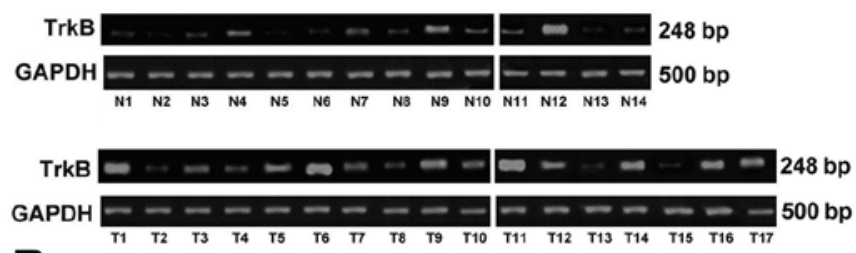

B
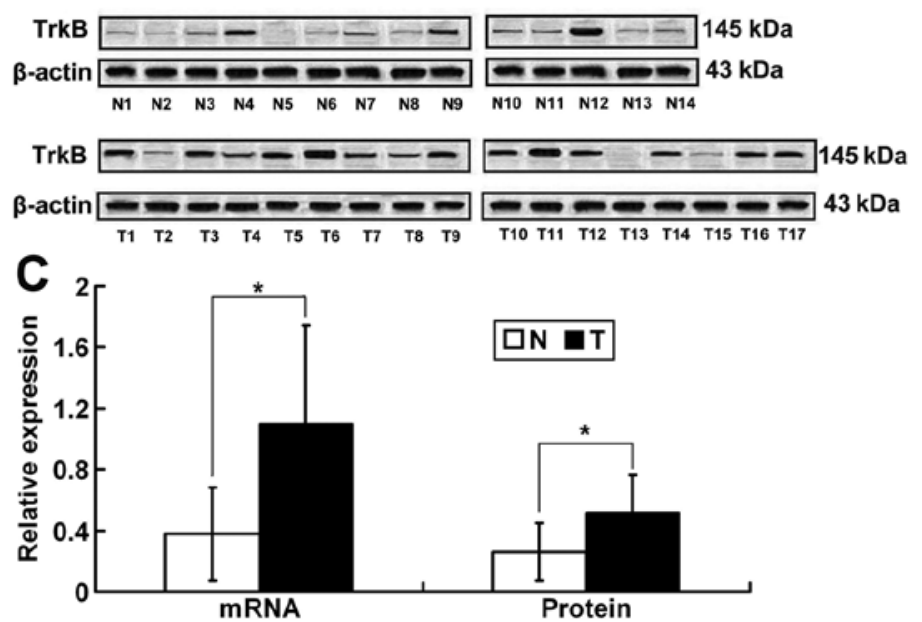

Figure 2. Expression levels of TrkB mRNA and protein were upregulated in NPC tissues. (A) RT-PCR results (N1-14, noncancerous nasopharyngeal tissues; T1-17, NPC tissues). (B) Western blot analysis results (N1-14, noncancerous nasopharyngeal tissues; T1-17, NPC tissues). (C) Relative expression levels of TrkB mRNA and protein in NPC (T) and noncancerous nasopharyngeal (N) tissues. The expression levels of TrkB mRNA and protein are shown relative to the levels of GAPDH mRNA and $\beta$-actin protein, respectively. The Student's t-test demonstrated that the expression levels of TrkB mRNA and protein in NPC tissues were significantly higher than the levels in noncancerous nasopharyngeal tissues (mRNA, $\mathrm{P}=0.000<0.01 ; \mathrm{protein}, \mathrm{P}=0.003<0.01$ ). 
Table III. Multivariate analysis of Cox model analysis for disease-free survival and overall survival.

\begin{tabular}{|c|c|c|c|c|c|c|}
\hline \multirow[b]{2}{*}{ Variables } & \multicolumn{3}{|c|}{ Disease-free survival } & \multicolumn{3}{|c|}{ Overall survival } \\
\hline & Risk ratio & 95\% Confidence interval & P-value & Risk ratio & 95\% Confidence interval & P-value \\
\hline Gender (male/female) & 0.615 & $0.263-1.438$ & 0.262 & 0.517 & $0.195-1.370$ & 0.184 \\
\hline Age $(\leq 50 />50$ years $)$ & 1.135 & $0.631-2.040$ & 0.672 & 1.295 & $0.682-2.458$ & 0.430 \\
\hline $\begin{array}{l}\text { Histological type } \\
\text { (keratinizing/non-keratinizing) }\end{array}$ & 3.072 & $0.374-25.246$ & 0.296 & 2.142 & $0.250-18.371$ & 0.487 \\
\hline $\mathrm{T}$ classification $(\mathrm{T} 1+\mathrm{T} 2 / \mathrm{T} 3+\mathrm{T} 4)$ & 1.340 & $0.731-2.457$ & 0.343 & 1.433 & $0.735-2.793$ & 0.291 \\
\hline $\mathrm{N}$ classification $(\mathrm{N} 0+\mathrm{N} 1 / \mathrm{N} 2+\mathrm{N} 3)$ & 6.609 & $1.539-28.391$ & 0.011 & 5.216 & $1.193-22.807$ & 0.028 \\
\hline M classification (M0/M1) & 2.544 & $1.125-5.751$ & 0.025 & 2.359 & $1.005-5.538$ & 0.049 \\
\hline Clinical stage (I+II/III+IV) & 0.455 & $0.057-3.668$ & 0.460 & 0.369 & $0.044-3.087$ & 0.357 \\
\hline Trkb expression (high/low) & 1.600 & $1.040-2.461$ & 0.033 & 1.863 & $1.139-3.048$ & 0.013 \\
\hline
\end{tabular}

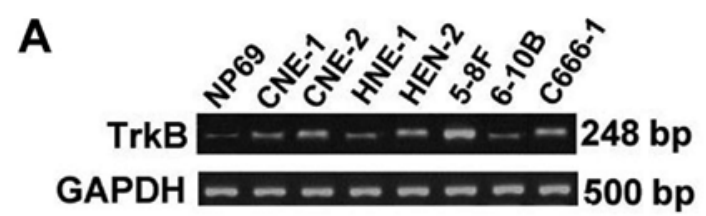

B

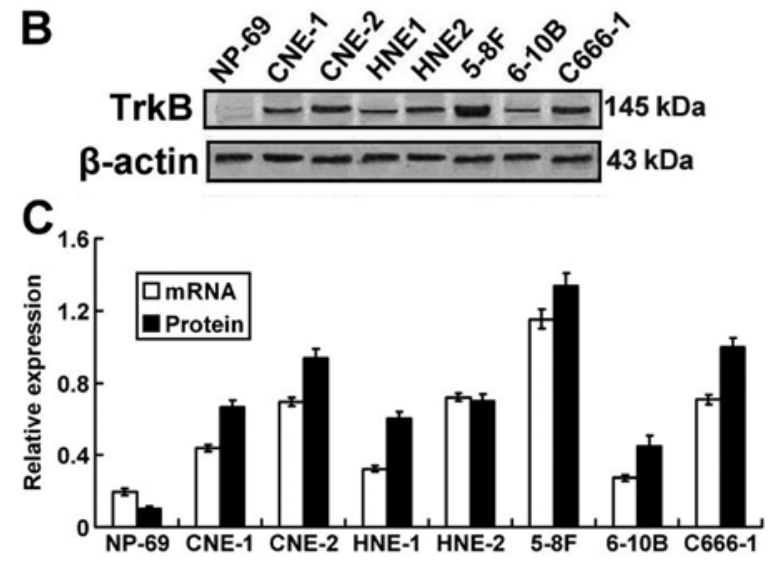

Figure 3. Expression levels of TrkB mRNA and protein were upregulated in the NPC cell lines. (A) RT-PCR results of the 7 NPC cell lines and the nontransformed nasopharyngeal epithelium cell line (NP-69). (B) Western blot results of the 7 NPC cell lines and the NP-69 cell line. (C) Relative expression levels of TrkB mRNA and protein in the 7 NPC cell lines and the NP-69 cell line. The expression levels of TrkB mRNA and protein are shown relative to the levels of GAPDH mRNA and $\beta$-actin protein, respectively.

patients in the high expression group had both reduced overall survival and disease-free survival when compared with patients in the low expression group (overall survival rate, 48.8 vs. $73.8 \%, \mathrm{P}=0.001<0.05$; disease-free survival rate, 41.9 vs. $66.2 \%, \mathrm{P}=0.001<0.05$ ) (Fig. 4). In the multivariate Cox regression analysis, high TrkB expression [risk ratio $(\mathrm{RR})=1.863$, $\mathrm{P}=0.013]$, poor $\mathrm{N}$ classification $(\mathrm{R}=5.216, \mathrm{P}=0.028)$ and $\mathrm{M}$ classification $(\mathrm{RR}=2.359, \mathrm{P}=0.049)$ were identified as independent prognostic factors for overall survival. Moreover, these 3 clinicopathologic parameters were identified as independent prognostic factors for disease-free survival (TrkB expression: $\mathrm{RR}=1.600$ and $\mathrm{P}=0.033 ; \mathrm{N}$ classification: $\mathrm{RR}=6.609$ and $\mathrm{P}=0.011 ; \mathrm{M}$ classification: $\mathrm{RR}=2.544$ and $\mathrm{P}=0.025)$. The other clinicopathological parameters including age, gender, histological type, T classification and AJCC clinical stage that were examined did not provide any independent prognostic information (Table III).

\section{Discussion}

In the present study, TrkB mRNA and protein levels were identified to be significantly upregulated in NPC tissues and cell lines. Its expression was strongly correlated with enhanced tumor invasion, advanced clinical stage and positive lymph node status.

Environmental factors, genetic susceptibility and EBV infection play roles in the development of NPC. NPC is rare in most regions of the world; however, it is a common cancer in Southern China, particularly in Guangdong and Guangxi and Hunan Provinces. Radiotherapy with or without chemo- 

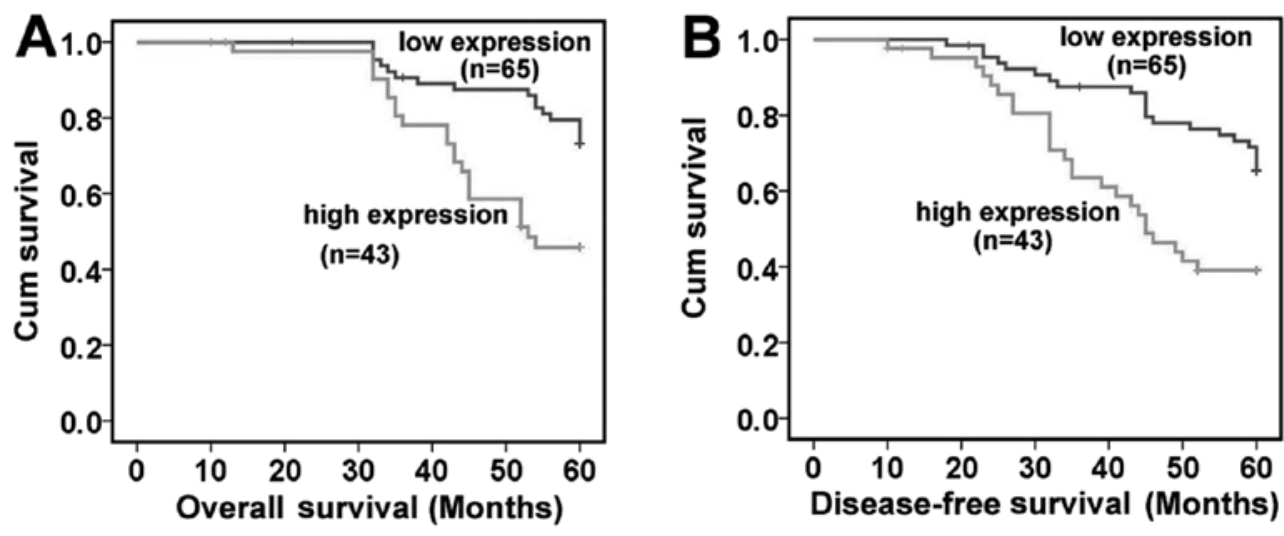

Figure 4. Estimated disease-free and overall survival according to the expression of TrkB in 108 patients with NPC. Kaplan-Meier patient survival analysis indicated that patients with NPC in the high TrkB expression group had (A) reduced disease-free survival $(\mathrm{P}=0.001<0.05)$ and $(\mathrm{B})$ reduced overall survival $(\mathrm{P}=0.001<0.05)$ compared with patients in the low TrkB expression group.

therapy in NPC is the main treatment method. However, the treatment results of late stage NPC have been disappointing. Unfortunately, approximately $70 \%$ of newly diagnosed NPC patients are characterized as stage III or IV (16). The poor prognosis may be due to lymph node and distant metastasis (16-18). The mechanisms at the molecular level of NPC invasion and metastasis are still not completely clear.

Receptor tyrosine kinases (RTKs) have been shown to regulate normal cellular processes. Certain RTKs were found to be overexpressed in malignant tissue (19). Thus, these RTKs were characterized as oncogenes. Preclinical trials of target therapies on certain RTKs showed promising results (20). TrkB is one of the RTKs and regulates various cellular processes in the nervous system, including neuronal survival, differentiation, maintenance and migration. Support for a role of TrkB in human tumors initially came from studies on its expression pattern in neuroblastoma. TrkB overexpression is common in neuroblastoma, and is correlated with poor patient prognosis $(21,22)$. In our present study, the mRNA and protein expression levels of TrkB were significantly higher in NPC samples than these levels in the normal nasopharyngeal tissues. In two independent studies on pancreatic ductal adenocarcinomas, elevated TrkB expression was noted in tumor cells when compared with that in the surrounding normal tissue in $63 \%$ of 47 and $50 \%$ of 54 cases examined, respectively $(8,23)$. Zhang et al (9) analyzed TrkB expression in 161 gastric carcinoma patients by immunohistochemistry and western blot analysis, and a high level of TrkB expression was observed in well-differentiated gastric carcinoma subtypes. In the present study, both mRNA and protein expression of TrkB was also upregulated in 7 NPC cell lines when compared to these levels in a nontransformed nasopharyngeal epithelium cell line. The mechanisms at the molecular level of the overexpression of TrkB in NPC is not clear, but the present results that both mRNA and protein expression of TrkB was upregulated in NPC samples and cell lines indicate a pre-transcriptional mechanism in NPC.

TrkB has been demonstrated to be involved in the process of invasion and metastasis in several other types of malignancies, including lung, thyroid, breast, pancreatic and prostate cancers, lymphoma and Wilms' tumor (24). Consistent with previous reports, among the clinicopathologic factors examined, results from our study revealed a significant asso- ciation between TrkB expression and tumor size, lymph node metastasis and clinical stage indicating the close association between TrkB expression and NPC progression and metastasis. Additionally, as compared to other NPC cell lines (CNE-1, CNE-2, HNE-1, HEN-2, 6-10B and C666-1), the 5-8F cell line, which has high metastatic potential (14), displayed the highest TrkB mRNA and protein expression levels. This result demonstrated a potential association between TrkB overexpression and a malignant phenotype such as metastatic ability. Recently, TrkB has been reported to increase the invasive and metastatic abilities of many types of cancers by suppressing anoikis (25). Moreover, studies with head and neck cancer cell lines revealed that in vitro stimulation with BDNF, the ligand for TrkB, increased the migratory and invasive capabilities of head and neck cancer cells by altering the expression of molecular mediators of epithelial-to-mesenchymal transition (EMT) (26). Our present results and previous reports provide evidence that aberrant TrkB signaling is sufficient to induce tumorigenesis as well as invasion and metastasis.

The Kaplan-Meier method was used to assess the role of TrkB expression levels to predict the prognosis of patients with NPC. The result demonstrated that NPC patients with high TrkB expression were significantly associated with a shorter disease-free survival and overall survival. To determine whether the TrkB expression level could serve as an independent prognostic factor for patients with NPC, we subsequently constructed a Cox regression model. In multivariate Cox regression analysis including 8 clinicopathological parameters, such as patient gender, age, histological type, tumor size, lymph node metastasis, distant metastasis, AJCC clinical stage and TrkB expression level, the TrkB expression level was verified as an independent prognostic indicator for patients with NPC. Our present results were consistent with several other reports concerning other tumors. Okamura et al (27) evaluated TrkB and BDNF expression in non-small cell lung cancer patient samples by immunohistochemistry, and found that expression of TrkB and BDNF was associated with poor prognosis in non-small cell lung cancer patients. A research study of 102 colorectal cancer patients showed that patients with high TrkB mRNA expression in clinical samples had a significantly poorer prognosis relative to those with low TrkB levels (28). 
In conclusion, our present study revealed the possible involvement of TrkB in the induction of tumor invasion and lymph node metastases and demonstrated that elevated TrkB expression levels were associated with the progression and poor prognosis of NPC. The results suggest that TrkB may be used as a new prognostic marker for NPC. Moreover, since TrkB special inhibitors have already been developed (29), specific drug-mediated inactivation of TrkB may provide a novel method with which to have an effective therapeutic impact on poor clinical stage NPC with overexpressed or mutated TrkB.

\section{Acknowledgements}

We thank Daiqiang Li and Songqing Fang (Department of Pathology, The Second Xiangya Hospital, Central South University, Changsha, China) for their evaluation of the clinical samples.

\section{References}

1. Tang L, Mao Y, Liu L, et al: The volume to be irradiated during selective neck irradiation in nasopharyngeal carcinoma: analysis of the spread patterns in lymph nodes by magnetic resonance imaging. Cancer 115: 680-688, 2009.

2. Agulnik M and Siu LL: State-of-the-art management of nasopharyngeal carcinoma: current and future directions. Br J Cancer 92: 799-806, 2005.

3. Caponigro F, Longo F, Ionna F and Perri F: Treatment approaches to nasopharyngeal carcinoma: a review. Anticancer Drugs 21: 471-477, 2010

4. Chang ET and Adami HO: The enigmatic epidemiology of nasopharyngeal carcinoma. Cancer Epidemiol Biomarkers Prev 15: 1765-1777, 2006

5. Brodeur GM, Minturn JE, Ho R, et al: Trk receptor expression and inhibition in neuroblastomas. Clin Cancer Res 15: 3244-3250, 2009.

6. Schramm A, Schulte JH, Astrahantseff K, et al: Biological effects of TrkA and TrkB receptor signaling in neuroblastoma. Cancer Lett 228: 143-153, 2005.

7. Ricci A, Greco S, Mariotta S, et al: Neurotrophins and neurotrophin receptors in human lung cancer. Am J Respir Cell Mol Biol 25: 439-446, 2001.

8. Sclabas GM, Fujioka S, Schmidt C, et al: Overexpression of tropomysin-related kinase B in metastatic human pancreatic cancer cells. Clin Cancer Res 11: 440-449, 2005.

9. Zhang Y, Fujiwara Y, Doki Y, et al: Overexpression of tyrosine kinase B protein as a predictor for distant metastases and prognosis in gastric carcinoma. Oncology 75: 17-26, 2008.

10. Yu X, Liu L, Cai B, He Y and Wan X: Suppression of anoikis by the neurotrophic receptor TrkB in human ovarian cancer. Cancer Sci 99: 543-552, 2008.

11. Yu Y, Zhang S, Wang X, Yang Z and Ou G: Overexpression of TrkB promotes the progression of colon cancer. APMIS 118: $188-195,2010$
12. Yao KT, Zhang HY, Zhu HC, et al: Establishment and characterization of two epithelial tumor cell lines (HNE-1 and HONE-1) latently infected with Epstein-Barr virus and derived from nasopharyngeal carcinomas. Int J Cancer 45: 83-89, 1990.

13. Cheung ST, Huang DP, Hui AB, et al: Nasopharyngeal carcinoma cell line (C666-1) consistently harbouring Epstein-Barr virus. Int J Cancer 83: 121-126, 1999.

14. Li J, Fan Y, Chen J, Yao KT and Huang ZX: Microarray analysis of differentially expressed genes between nasopharyngeal carcinoma cell lines 5-8F and 6-10B. Cancer Genet Cytogenet 196: 23-30, 2010.

15. Tsao SW, Wang X, Liu Y, et al: Establishment of two immortalized nasopharyngeal epithelial cell lines using SV40 large T and HPV16E6/E7 viral oncogenes. Biochim Biophys Acta 1590: $150-158,2002$.

16. Chan AT, Teo PM, Ngan RK, et al: Concurrent chemotherapy-radiotherapy compared with radiotherapy alone in locoregionally advanced nasopharyngeal carcinoma: progression-free survival analysis of a phase III randomized trial. J Clin Oncol 20: 2038-2044, 2002

17. Xu T, Hu C, Wang X and Shen C: Role of chemoradiotherapy in intermediate prognosis nasopharyngeal carcinoma. Oral Oncol 47: 408-413, 2011.

18. Chen MK, Yang SF, Lai JC, et al: Expression of bcl-2 correlates with poor prognosis and modulates migration of nasopharyngeal carcinoma cells. Clin Chim Acta 411: 400-405, 2010.

19. Casaletto JB and McClatchey AI: Spatial regulation of receptor tyrosine kinases in development and cancer. Nat Rev Cancer 411: 387-400, 2012.

20. Shimizu M, Adachi S, Masuda M, Kozawa O and Moriwaki H: Cancer chemoprevention with green tea catechins by targeting receptor tyrosine kinases. Mol Nutr Food Res 55: 832-843, 2011.

21. Edsjö A, Lavenius E, Nilsson H, et al: Expression of TrkB in human neuroblastoma in relation to MYCN expression and retinoic acid treatment. Lab Invest 83: 813-823, 2003.

22. Eggert A, Ikegaki N, Liu XG and Brodeur GM: Prognostic and biological role of neurotrophin-receptor TrkA and TrkB in neuroblastoma. Klin Padiatr 212: 200-205, 2000.

23. Miknyoczki SJ, Lang D, Huang L, Klein-Szanto AJ, Dionne CA and Ruggeri BA: Neurotrophins and Trk receptors in human pancreatic ductal adenocarcinoma: expression patterns and effects on in vitro invasive behavior. Int J Cancer 81: 417-427, 1999.

24. Desmet CJ and Peeper DS: The neurotrophic receptor TrkB: a drug target in anti-cancer therapy? Cell Mol Life Sci 63: 755-759, 2006.

25. Geiger TR and Peeper DS: The neurotrophic receptor TrkB in anoikis resistance and metastasis: a perspective. Cancer Res 65: 7033-7036, 2005

26. Kupferman ME, Jiffar T, El-Naggar A, et al: TrkB induces EMT and has a key role in invasion of head and neck squamous cell carcinoma. Oncogene 29: 2047-2059, 2010.

27. Okamura K, Harada T, Wang S, et al: Expression of TrkB and BDNF is associated with poor prognosis in non-small cell lung cancer. Lung Cancer 78: 100-106, 2012.

28. Fujikawa H, Tanaka K, Toiyama Y, et al: High TrkB expression levels are associated with poor prognosis and EMT induction in colorectal cancer cells. J Gastroenterol 47: 775-784, 2012.

29. Perez-Pinera P, Hernandez T, García-Suárez O, et al: The Trk tyrosine kinase inhibitor K252a regulates growth of lung adenocarcinomas. Mol Cell Biochem 295: 19-26, 2007. 